\title{
Nuclei beyond the drip line
}

\author{
J. N. De, ${ }^{*}$ X. Viñas, S. K. Patra, and M. Centelles \\ Departament d'Estructura i Constituents de la Matèria, Facultat de Física, Universitat de Barcelona, \\ Diagonal 647, E-08028 Barcelona, Spain
}

(Received 11 July 2001; published 18 October 2001)

\begin{abstract}
In the Thomas-Fermi model, calculations are presented for nuclei beyond the nuclear drip line at zero temperature. These nuclei are in equilibrium by the presence of an external gas, as may be envisaged in the astrophysical scenario. We find that there is a limiting asymmetry beyond which these nuclei can no longer be made stable.
\end{abstract}

DOI: 10.1103/PhysRevC.64.057306

Nuclear stability is limited by the drip lines. As one moves away from the valley of $\beta$ stability, e.g., by increasing the neutron excess, the neutron chemical potential $\mu_{n}$ that is negative for stable nuclei steadily decreases in magnitude until it becomes zero at the neutron drip line. Beyond this line the neutron chemical potential is positive and the system cannot hold the excess neutrons together, rendering itself unstable. Similar is the situation for nuclei with a proton excess. It was, however, found that it is possible to extend the drip lines $\left(\mu_{n}=0\right.$ or $\left.\mu_{p}=0\right)$ provided the nuclei have a temperature. In Ref. [1] this was achieved by assuming the nucleus to be a liquid drop in thermodynamical equilibrium (thermal and chemical) with a surrounding vapor that exerts an external pressure to stabilize the system, whereas in Ref. [2] it was done by assuming the nuclear drop to be in metastable equilibrium (pressure equal to zero).

In the context of the equation of state (EOS) of cold dense matter, equilibrium nuclei far beyond the nominal drip lines are to be considered. There the nuclei are immersed in a nucleonic gas, mostly of neutrons, which exerts pressure to keep the system stable. Nuclei in this scenario were first studied by Langer et al. [3] and later in a broader scope by Baym, Bethe and Pethick [4]. From a microscopic viewpoint, there are two basic concerns. One, a thermodynamically consistent treatment of the coexistence [5-8] of the two phases of nuclear matter (namely, the nuclear liquid and the gas enclosing the liquid), the other, a plausible description of the interface between the liquid and the gas $[9,10]$. As the density of the nuclear gas increases, the surface energy must decrease [7]. In a recent paper, it was shown by Centelles et al. [10] that for semi-infinite nuclear matter this problem can be circumvented by solving for the density profile in the subtraction procedure $[11,12]$, treating the density as the difference between its value in the nuclear-plus-gas phase and its value in the gas phase. The influence of the surrounding gas on the surface energy is then automatically taken into account. They invoked a mean-field model and showed how asymmetric infinite or semi-infinite nuclear matter with large neutron excess (positive pressure, positive neutron chemical potential) can remain in stable equilibrium at zero temperature with a surrounding gas of drip nucleons.

*On leave from the Variable Energy Cyclotron Centre, 1/AF, Bidhannagar, Calcutta 700 064, India.
PACS number(s): 21.60. $-\mathrm{n}, 21.10 .-\mathrm{k}, 21.65 .+\mathrm{f}$

In this Brief Report we generalize the idea of Ref. [10] to the case of finite nuclei at zero temperature and focus on the effect of the external gas on the structure and stability of the nuclei with large neutron excess. We may stress that our main aim is not to study the EOS of dense matter, but to give a better guide to understand the properties of isolated nuclei immersed in a nucleonic gas. This knowledge may afterwards be taken as input for a broader and careful study of the conditions under which nuclei can exist in neutron star matter. In order to treat the matter inside the nucleus with that outside in a consistent fashion, we look for density solutions for the nucleus-plus-gas phase and also for the gas phase alone, and isolate the nucleus from the surrounding gas by subtracting the latter from the former. This method formally is similar to that of finding solutions to the coexistence between a hot nucleus and the evaporated nucleons around it $[11,12]$. We employ the Thomas-Fermi formalism, which has earlier been used as a convenient tool [13-15] to understand the EOS of dense nuclear matter. Here we resort to the Thomas-Fermi method restructured for the subtraction procedure [16] to get the density solutions as mentioned earlier. For the nuclear force, we choose the Skyrme interaction $\mathrm{SkM}^{*}$ [17]. In doing the calculations beyond the drip lines we find that the nuclear asymmetry $I$ cannot be increased arbitrarily (we define the asymmetry of the finite nucleus as $I=(N-Z) / A)$. There is a limiting asymmetry beyond which the thermodynamic equilibrium conditions cannot be met.

The subtraction procedure in the Thomas-Fermi prescription has been described in detail in Refs. $[10,16]$; we present here only the working equations. The method is based on the existence of two solutions to the Thomas-Fermi equations, one corresponding to the nucleus phase with the surrounding gas (NG) and the other corresponding to the gas $G$ alone. The densities in the NG and the $G$ phase are obtained by solving the coupled equations

$$
\begin{gathered}
\left(3 \pi^{2}\right)^{2 / 3} \frac{\hbar^{2}}{2 m_{\tau}^{*}} \rho_{N G}^{2 / 3}+V_{N G}^{\tau}+V_{N G}^{c}\left(\rho_{N G}, \rho_{G}\right)=\mu^{\tau}, \\
\left(3 \pi^{2}\right)^{2 / 3} \frac{\hbar^{2}}{2 m_{\tau}^{*}} \rho_{G}^{2 / 3}+V_{G}^{\tau}+V_{G}^{c}\left(\rho_{N G}, \rho_{G}\right)=\mu^{\tau} .
\end{gathered}
$$

The density of the nucleus is then given by 


$$
\rho(r)=\rho_{N G}(r)-\rho_{G}(r)
$$

so that

$$
A^{\tau}=\int\left[\rho_{N G}^{\tau}(r)-\rho_{G}^{\tau}(r)\right] d^{3} r,
$$

where $A^{\tau}$ is the neutron or proton number $(N$ or $Z$ ) of the nucleus in question. In Eqs. (1) and (2), $\mu^{\tau}$ is the chemical potential; it defines the nucleus as

$$
\begin{aligned}
\mu^{\tau}= & \frac{1}{A^{\tau}}\left\{\int \left[\left(3 \pi^{2}\right)^{2 / 3} \frac{\hbar^{2}}{2 m_{\tau}^{*}} \rho_{N G}^{2 / 3}(r)+V_{N G}^{\tau}(r)\right.\right. \\
& \left.+V_{N G}^{c}(r)\right] \rho_{N G}(r) d^{3} r-\int\left[\left(3 \pi^{2}\right)^{2 / 3} \frac{\hbar^{2}}{2 m_{\tau}^{*}} \rho_{G}^{2 / 3}(r)\right. \\
& \left.\left.+V_{G}^{\tau}(r)+V_{G}^{c}(r)\right] \rho_{G}(r) d^{3} r\right\} .
\end{aligned}
$$

Here $V^{\tau}$ is the nuclear part of the single-particle potential for either the NG or $G$ solution and $V^{c}$ is the Coulomb potential coupling both the solutions; $m_{\tau}^{*}$ used in Eqs. (1) and (2) are the density-dependent effective masses for each phase. The direct part of $V^{c}$ is the same for both phases and is given by

$$
V_{d}^{c}(r)=\frac{e^{2}}{2} \int\left[\rho_{N G}^{p}\left(r^{\prime}\right)-\rho_{G}^{p}\left(r^{\prime}\right)\right] g\left(r, r^{\prime}\right) d^{3} r^{\prime}
$$

with

$$
g\left(r, r^{\prime}\right)=\left[\left(r+r^{\prime}\right)-\left|r-r^{\prime}\right|\right] /\left(r r^{\prime}\right) .
$$

The Coulomb potentials in the NG and $G$ phases differ only through the exchange terms: $-e^{2}(3 / \pi)^{1 / 3}\left(\rho_{N G, G}^{p}\right)^{1 / 3}$. They, however, have the same asymptotic value. Equations (1) through (6) are solved self-consistently in an iterative manner to yield the densities $\rho_{N G}(r)$ and $\rho_{G}(r)$. In neutron-rich nuclei the proton density in the gas phase is $\rho_{G}^{p}(r)=0$, similarly in proton-rich nuclei, there are no neutrons in the gas phase. Solutions obtained in this manner ensure mechanical and chemical equilibrium between the nuclear phase and the drip phase.

We have done calculations for the isotopes of four systems, namely, those of $\mathrm{Ca}, \mathrm{Zr}, \mathrm{Sn}$, and $\mathrm{Pb}$. This covers a broad range of charges. As one adds more neutrons to a system with fixed $Z$, the neutron chemical potential (which is negative for a stable nucleus) increases until it crosses zero meaning thereby that further addition of neutrons would not allow them to be part of a bound system causing them to drip (the neutron drip line). Similarly, removal of neutrons for a system with fixed charge increases the proton chemical potential for the system until it reaches the proton drip line when the system becomes unstable.

If these nuclei are, however, surrounded by a gas of nucleons as may well be the case in the astrophysical scenario, say after a supernova explosion, the excess pressure given by the gas (drip phase) may stabilize them and then one may reach the stability limits beyond the nominal drip line. For ex-

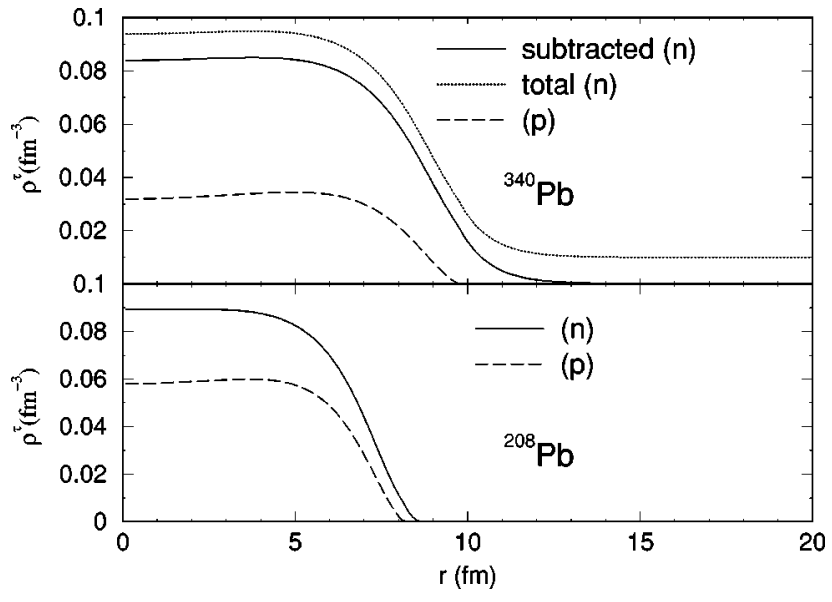

FIG. 1. The total and subtracted neutron and proton densities for ${ }^{340} \mathrm{~Pb}$ are shown in the upper panel. In this nucleus, there are no protons in the drip phase. In the lower panel, the densities for ${ }^{208} \mathrm{~Pb}$ are displayed.

ample, ${ }^{68} \mathrm{Ca}$ is the neutron drip-line nucleus in our calculation, but with a drip phase surrounding the nucleus one can reach ${ }^{88} \mathrm{Ca}$ as a stable system. The density of the drip phase (in this case that of the drip neutrons) increases with the increasing number of neutrons in the nucleus. Similarly, ${ }^{35} \mathrm{Ca}$ is the proton drip-line nucleus. One can, however, go further below in mass to ${ }^{25} \mathrm{Ca}$ with a surrounding proton gas. The drip phase of the protons is assumed to be charge neutral [12]; in the astrophysical context the proton gas is neutralized by the electrons pervading the gas. The external gas does not contribute to the Coulomb energy, the Coulomb potential $V^{c}$ is calculated from the subtracted proton density that is exactly the proton density of the isolated nucleus.

In Fig. 1, the density distributions of ${ }^{340} \mathrm{~Pb}$ are shown. The nucleus has 63 more neutrons than the drip-line nucleus ${ }^{277} \mathrm{~Pb}$ as found in our calculation. We display the density of the nucleus plus gas and also the subtracted density. The subtracted density is found to be independent of the size of the box in which the calculations are done. The neutron dripphase density is seen to grow to more than one-tenth of the central neutron density. There are no protons in this drip phase. For comparison, the densities of the nucleus ${ }^{208} \mathrm{~Pb}$ are also displayed. One can easily discern the thicker neutron skin in ${ }^{340} \mathrm{~Pb}$ and also the effect of the asymmetry potential in pulling the proton distribution outwards from the origin as compared to that in ${ }^{208} \mathrm{~Pb}$. The central proton density is decreased to almost half of its value in ${ }^{208} \mathrm{~Pb}$. In Fig. 2, the density distributions for the very proton-rich nucleus ${ }^{140} \mathrm{~Pb}$ are shown. Here the drip phase consists only of protons. It is polarized because of the influence of the Coulomb field of the nucleus on the surrounding gas as seen in the case of evaporated protons from a hot nucleus $[11,12]$. In all cases, the nucleus-plus-gas solution coincides with the gas solution asymptotically at a large distance for both neutrons and protons.

In the calculations on infinite or semi-infinite nuclear matter in equilibrium with the drip phase, it was observed in Refs. $[5,9,10]$ that the asymmetry could be increased arbitrarily till the densities and asymmetries of both phases 


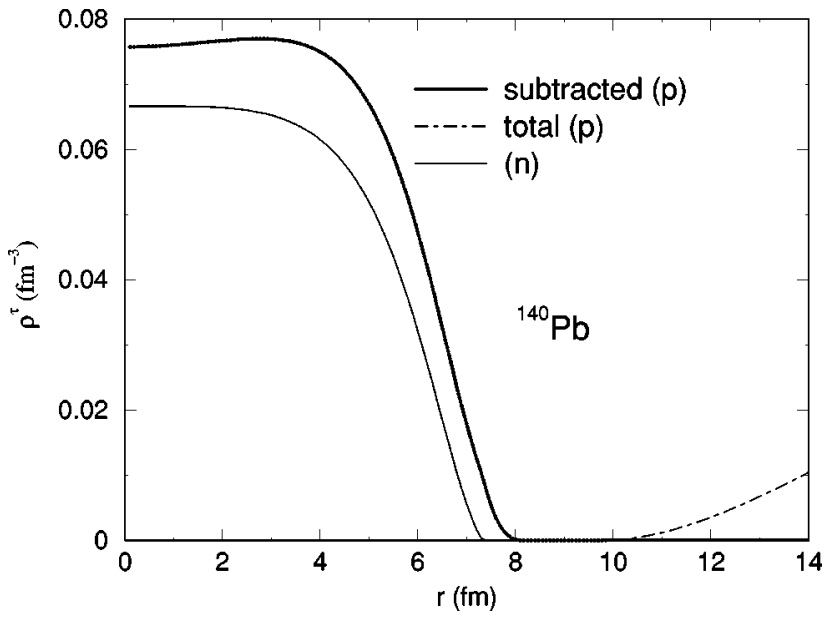

FIG. 2. The total and subtracted neutron and proton densities for the nucleus ${ }^{140} \mathrm{~Pb}$. In this nucleus, there are no neutrons in the drip phase. In the nuclear interior (up to $\sim 10 \mathrm{fm}$ ), the total and subtracted proton densities are indistinguishable, beyond this, the polarization of the proton density in the drip phase is evident.

merged with each other. In the self-consistent calculations in finite systems, we, however, observe that one cannot add or remove neutrons from a nucleus arbitrarily. Beyond a certain point, further addition or removal creates instabilities in the system and no solution to the Thomas-Fermi problem can be found. We call this the limiting asymmetry. Establishing full chemical equilibrium between the nuclear phase and the drip phase becomes harder in these conditions, and beyond the limiting asymmetry it is no longer possible. This type of instabilities has a remarkable similarity to those found in the calculation of limiting temperatures for hot nuclei [11,12]. As a function of the charge $Z$ of the nucleus, the limiting asymmetry along with the asymmetry at the drip lines are plotted in Fig. 3. On the neutron-rich side, they are found to be almost constant in the charge range we consider. On the neutron-deficient side, their magnitudes decrease with increasing charge. The wider gap between the nominal asymmetry (proton drip line) and the limiting asymmetry, due to

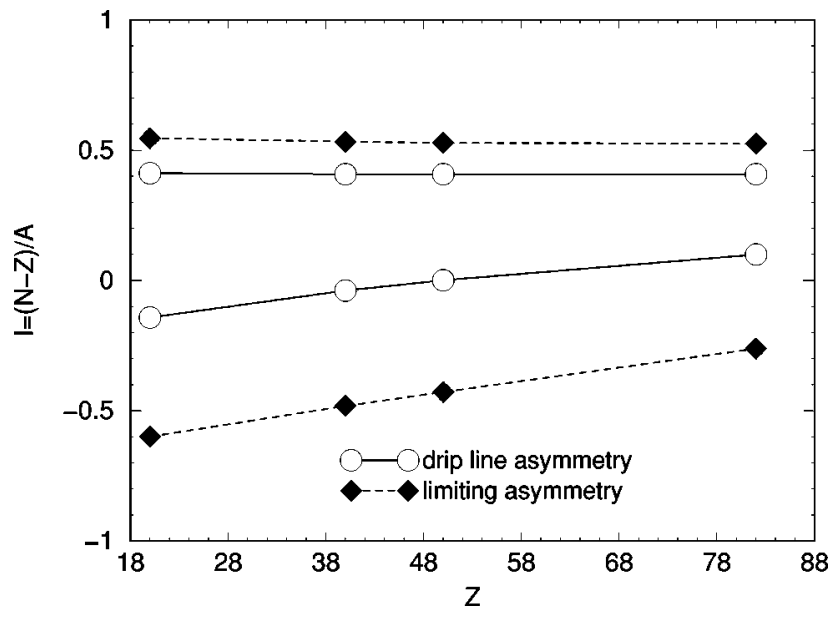

FIG. 3. The limiting and drip line asymmetries as a function of the charge number of nuclei.

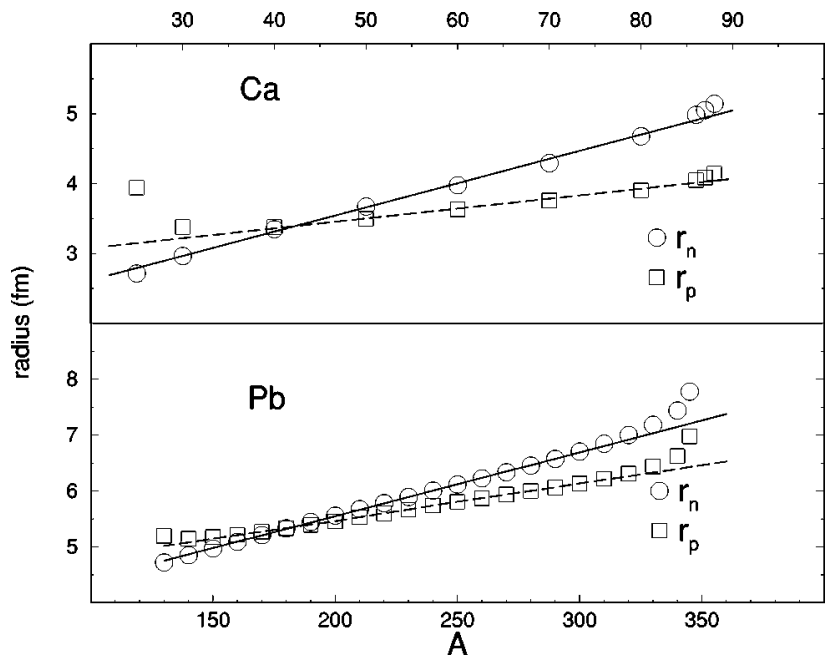

FIG. 4. rms radii of neutrons and protons for the isotopes of $\mathrm{Ca}$ (upper panel) and $\mathrm{Pb}$ (lower panel). The open circles and squares refer to the calculated neutron and proton radii as indicated. The solid and the dashed lines refer to linear fits of the radii to the mass number $A$ (the nuclei at the edges have been excluded from the fit).

the Coulomb repulsion is easily discernible. In Fig. 4, the rms radii of neutrons and protons for the $\mathrm{Ca}$ and $\mathrm{Pb}$ isotopes are displayed. One sees the growth of the neutron or proton skins with positive or negative asymmetry. One further finds that except for the nuclei near the edges of limiting asymmetry, the rms radii scale perfectly with $A$, the mass number. On both sides of the mass scale, the sudden rise in the neutron or proton radius points to the onset of instability.

To conclude, we have done calculations in a suitably modified Thomas-Fermi model for nuclei beyond the drip lines, when the nuclei are immersed in a nucleonic fluid. In such a prescription, no extra care is needed to treat the interface region of the nucleus and the surrounding fluid or to isolate the nucleus from the environment. From the numerical calculations, a limiting asymmetry beyond which nuclei even within the gaseous environment cannot exist is found; the delicate balance between the Coulomb force and the diluted surface tension with increasing asymmetry and increasing density of the environment very likely plays the crucial role there. We have not gone into the extensions of the Thomas-Fermi scheme, have not taken shell effects into consideration, and have worked with the $\mathrm{SkM}^{*}$ interaction whose validity at large asymmetry is not unquestionable. Sophistications in the approach or the use of different interactions may change the results somewhat quantitatively, but the qualitative features of the physics which emerge from our calculations, we believe, will remain unchanged.

The authors would like to acknowledge support from the DGICYT (Spain) under Grant No. PB98-1247 and from DGR (Catalonia) under Grant No. 1998SGR-00011. J.N.D. and S.K.P. thank the Spanish Ministry of Education for financial support with Grant Nos. SAB1999-0229 and SB97OL174874 and the Departament d'Estructura i Constituents de la Matèria of the University of Barcelona for the kind hospitality extended to them. 
[1] J. Besprosvany and S. Levit, Phys. Lett. B 217, 1 (1989).

[2] J. N. De, D. Bandyopadhyay, S. K. Samaddar, and N. Rudra, Nucl. Phys. A534, 294 (1991).

[3] W. D. Langer, L. C. Rosen, J. M. Cohen, and A. G. W. Cameron, Astrophys. Space Sci. 5, 529 (1969).

[4] G. Baym, H. Bethe, and C. J. Pethick, Nucl. Phys. A175, 225 (1971).

[5] J. M. Lattimer and D. G. Ravenhall, Astrophys. J. 223, 314 (1978).

[6] D. Q. Lamb, J. M. Lattimer, C. J. Pethick, and D. G. Ravenhall, Nucl. Phys. A360, 459 (1981).

[7] J. M. Lattimer, C. J. Pethick, D. G. Ravenhall, and D. Q. Lamb, Nucl. Phys. A432, 646 (1985).

[8] C. J. Pethick and D. G. Ravenhall, Annu. Rev. Nucl. Part. Sci. 45, 429 (1995).

[9] W. D. Myers, W. J. Swiatecki, and C. S. Wang, Nucl. Phys.
A436, 185 (1985).

[10] M. Centelles, M. Del Estal, and X. Viñas, Nucl. Phys. A635, 193 (1998)

[11] P. Bonche, S. Levit, and D. Vautherin, Nucl. Phys. A427, 278 (1984).

[12] P. Bonche, S. Levit, and D. Vautherin, Nucl. Phys. A436, 265 (1985).

[13] J. R. Buchler and Z. Barkat, Phys. Rev. Lett. 27, 48 (1971).

[14] R. Ogasawara and K. Sato, Prog. Theor. Phys. 70, 1569 (1983).

[15] M. Pi, X. Viñas, M. Barranco, A. Perez-Canyellas, and A. Polls, Astron. Astrophys., Suppl. Ser. 64, 439 (1986).

[16] E. Suraud, Nucl. Phys. A462, 109 (1987).

[17] M. Brack, C. Guet, and H.-B. Håkansson, Phys. Rep. 123, 276 (1985). 